\title{
Das Konzept «Waldbrand 2020» des Kantons Tessin
}

\author{
Aron Ghiringhelli \\ Gianni Boris Pezzatti \\ Marco Conedera
}

\author{
Ufficio forestale $1^{\circ}$ circondario del Cantone Ticino $(\mathrm{CH})^{*}$ \\ Eidgenössische Forschungsanstalt für Wald, Schnee und Landschaft $(\mathrm{CH})$ \\ Eidgenössische Forschungsanstalt für Wald, Schnee und Landschaft $(\mathrm{CH})$
}

\section{Das Konzept «Waldbrand 2020» des Kantons Tessin}

Der Kanton Tessin weist eine lange Tradition im Umgang mit Waldbränden auf. Das äussert sich darin, dass Waldbrände seit Langem systematisch dokumentiert und analysiert werden und ein Waldbrandmanagement existiert, das stetig verbessert wird mit dem Ziel, die Schutzfunktion des Waldes zu bewahren und die Bewohnbarkeit der Berggebiete zu sichern. Seit den 1970er-Jahren konnte das Waldbrandgeschehen im Tessin stark reduziert werden. Gründe dafür sind organisatorische und technische Verbesserungen bei der Brandbekämpfung (z.B. systematischer Helikoptereinsatz) und die Möglichkeit, bei hoher Waldbrandgefahr ein absolutes Feuerverbot im Freien aussprechen zu können. Die Ansprüche der Gesellschaft hinsichtlich Schutz von Menschenleben und Sachwerten nehmen aber stetig zu, und als Folge des Klimawandels dürfte auch das Waldbrandrisiko ansteigen. Als Reaktion darauf hat das Tessiner Kantonsforstamt in enger Zusammenarbeit mit dem kantonalen Feuerwehrverband und der Eidgenössischen Forschungsanstalt WSL vor gut zwei Jahren das Konzept «Waldbrand 2020» entwickelt. Es besteht aus den vier eng ineinander verwobenen Handlungsfeldern 1) Prävention, 2) technische und organisatorische Massnahmen, 3) Löschaktion und 4) Post-Brand-Management. Der Forstdienst ist zuständig für die Beurteilung der Brandgefahr, den Erlass von Feuerverboten, die Beratung der lokalen Behörden bei der Waldbewirtschaftung und der Planung der Löscheinrichtungen. Darüber hinaus definiert er die Regeln für den Löscheinsatz per Helikopter, erfasst die Waldbrände für die Statistik und plant wo erforderlich Verbau- und Wiederinstandstellungsmassnahmen. Die eigentliche Waldbrandbekämpfung ist Aufgabe der Feuerwehr, die zuerst mit den Einheiten der Stadtfeuerwehr ausrückt, bei anspruchsvollen Löscheinsätzen aber die auf Waldbrände spezialisierte Bergfeuerwehr zu Hilfe ruft. Bei den Löscheinsätzen steht das Forstpersonal den Einsatzleitern der Feuerwehr beratend zur Seite. Wie eine erste Evaluation zeigt, ist das Konzept «Waldbrand 2020» zielführend. Einzelne Verbesserungsmöglichkeiten wurden erkannt (z.B. Vervollständigung des Löschbeckennetzes, bessere Koordination zwischen Löschaktionen aus der Luft und am Boden, Verstärkung der spezialisierten Bergfeuerwehr) und befinden sich in Umsetzung.

Keywords: fire management, fire prevention, fire pre-suppression, post-fire restoration, fire danger rating doi: $10.3188 /$ szf. 2019.0242

* Piazza S. Franscini 3b, CH-6760 Faido, E-Mail aron.ghiringhelli@ti.ch

$\mathrm{D}$ ie Alpensüdseite ist historisch gesehen die am stärksten von Waldbränden betroffene Region der Schweiz. Sie weist auch eine lange Tradition im Umgang mit Waldbränden auf. Im Kanton Tessin äussert sich dies darin, dass Waldbrände systematisch dokumentiert und analysiert werden (Ceschi 1975/76, Conedera et al 1996, Conedera \& Pezzatti 2005) und ein Waldbrandmanagement existiert, das aufgrund der gesammelten Erfahrungen stetig verbessert wird (Conedera et al 2004). Dessen Hauptziel ist es, die Schutzfunktion des Waldes zu bewahren und so die Unversehrtheit und die Bewohnbarkeit der Alpentäler zu sichern (SFC 2007). Anders als beispielsweise im Mittelmeergebiet oder in Kalifornien, wo das Feuer eine unmit- telbare Bedrohung für die Bevölkerung darstellt (Kocher \& Butsic 2017), sind die Waldbrände in den Alpen in erster Linie eine Gefahr für den Wald und seine Schutzfunktion. Jedoch sind die Ansprüche der Gesellschaft an den Schutz von Bevölkerung, Siedlungen und Infrastrukturen gegenüber früher enorm gestiegen. Dazu kommt, dass aufgrund des Klimawandels trockenere und wärmere Sommer sowie schneearme Winter prognostiziert werden (NCCS 2018), die die Waldbrandgefahr in Zukunft auf ein noch nie dagewesenes und schwer abschätzbares Niveau ansteigen lassen.

Um dieser düsteren Perspektive entgegenzuwirken, hat das Tessiner Kantonsforstamt in enger Zusammenarbeit mit dem kantonalen Feuerwehr- 
a) Anzahl Brände

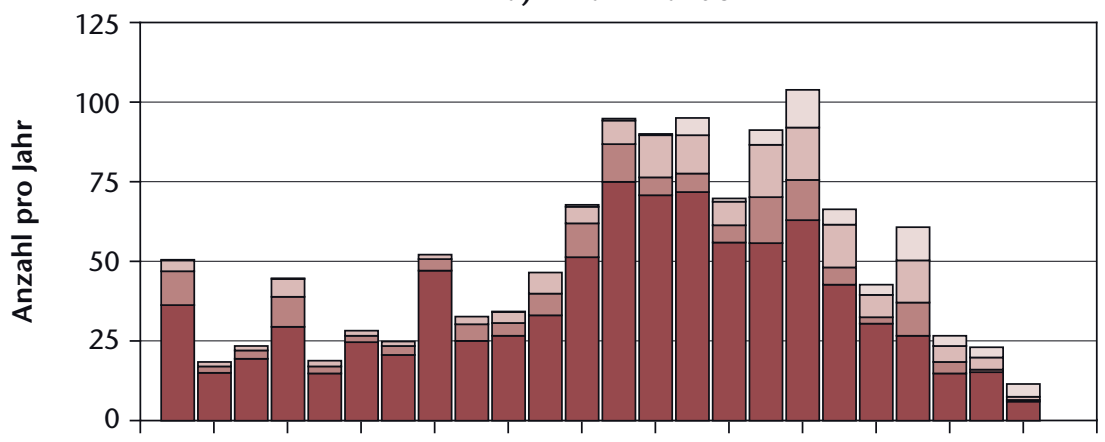

b) Brandfläche

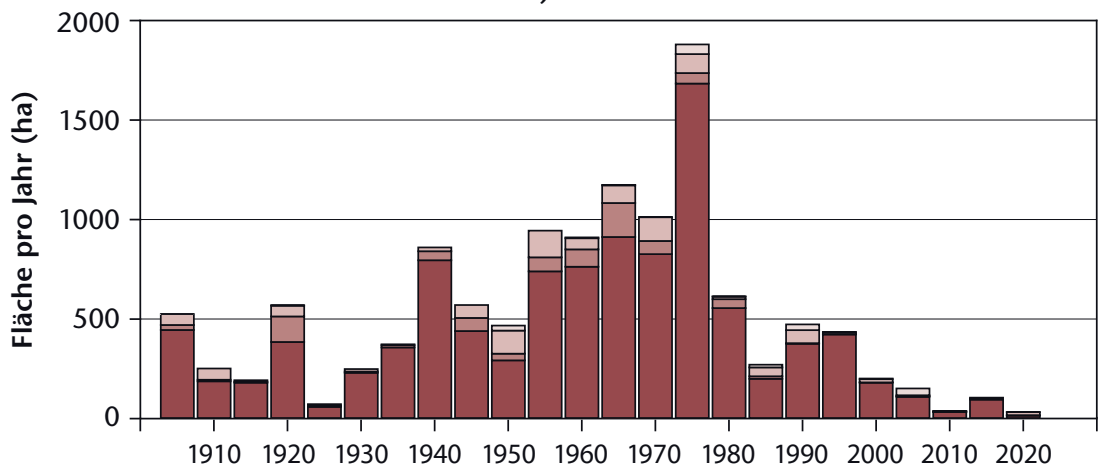

$\square$ Winter - vom Mensch verursacht $\quad \square$ Sommer - unbekannte Ursache $\square$ Sommer - vom Mensch verursacht $\square$ Sommer - Blitzschlagbrände

Abb 1 Entwicklung der Anzahl Brände (a) und der Brandfläche (b) pro Jahr im Kanton Tessin in den letzten 120 Jahren. Die Daten sind in 5-Jahres-Perioden gruppiert.

verband das kantonale Konzept «Waldbrand 2020» entwickelt (SFC 2017). Das am 18. Januar 2017 vom Regierungsrat gutgeheissene Konzept orientiert sich am Prinzip des integralen Risikomanagements, das im Umgang mit Naturgefahren in der Schweiz heute üblich ist (Planat 2018). Dabei ist das oberste Ziel, das Waldbrandrisiko unter Berücksichtigung der zur Verfügung stehenden Mittel dauernd auf ein für die Gesellschaft akzeptables Niveau zu begrenzen. Das Konzepts umfasst im Wesentlichen vier Handlungsfelder: Die Optimierung der Investitionen in die Waldbrandprävention, die Feuerwehrorganisation, die Waldbrandbekämpfung sowie die Wiederinstandstellung der Brandflächen mit dem Ziel, die Schutzfunktion kontinuierlich, langfristig und möglichst flächendeckend zu erhalten.

In diesem Beitrag stellen wir die wesentlichen Inhalte des kantonalen Konzepts «Waldbrand 2020» vor und ziehen Bilanz über die ersten zwei Jahre seit Inkrafttreten.

\section{Das Waldbrandgeschehen im Kanton Tessin}

Der Kanton Tessin hat eine Fläche von 274000 ha und ist zu 52\% bewaldet (Cioldi et al 2010). Die dank viel Niederschlag bis in tiefe Lagen üppige Vegetation wird während der langen Trockenperioden zu leicht entzündbarem Brandgut. Daraus ergeben sich ideale Bedingungen für Waldbrände, insbesondere bei Nordföhn (Cesti 1990).

Die Anfälligkeit des Kantons Tessin für Waldbrand wurde in den Jahren 1960 bis 1990 deutlich, als sich die sozioökonomischen Verhältnisse rasch änderten. Die traditionellen landwirtschaftlichen Aktivitäten gingen zurück, die Streunutzung wurde gar ganz eingestellt und die Holznutzung nahm stark ab. Dafür nahm die Waldfläche zu. Dies führte zu einem dramatischen Anstieg der Waldbrände (Abbildung 1): Waren zwischen 1931 und 1960 noch jährlich 38.3 ( \pm 24.9 ) Brände zu verzeichnen, waren es zwischen 1961 und 1990 jährlich 92.5 ( \pm 42.1), ohne dass sich die meteorologischen Verhältnisse wesentlich verändert hätten (Conedera \& Pezzatti 2005, Pezzatti et al 2013). Dank Beschlüssen, die das Feuermachen im Freien streng reglementierten oder gar verboten, reduzierte sich die Zahl der Brände in den 1990er-Jahren rapide (Abbildung 1a). Bereits ab den 1980er-Jahren konnten die Brandflächen erheblich verkleinert werden, dies dank organisatorischen und technischen Verbesserungen bei der Feuerwehr sowie dem systematischen Einsatz von Helikoptern bei den Löschaktionen (Abbildung 1b). Heutzutage (Periode 2000-2018) brechen im Kanton Tessin im Durchschnitt 38.6 ( \pm 17.9) Waldbrände pro Jahr aus, die eine Fläche von $116.0( \pm 161.0)$ ha erfassen. ${ }^{1}$ Die Situation variiert allerdings von Jahr zu Jahr und von Region zu Region stark (Conedera \& Pezzatti 2005).

Die heikelsten Monate bezüglich Waldbrand sind der März und der April (im Schnitt 32.9\% der Brände und $60.2 \%$ der Brandfläche in der Periode 2000-2018), weil dann die (dicke) Streuschicht unter der ruhenden Waldvegetation und die Witterung die Feuerentfachung und -ausbreitung begünstigen. Seit einigen Jahren nehmen aber auch die Brände im Sommer und insbesondere die durch Blitzschlag verursachten Brände zu (14.1\% aller Brände und 31.5\% der Sommerbrände in der Periode 2000-2018), eine Entwicklung, die sich mit der Klimaveränderung weiter zuspitzen könnte (Conedera et al 2006, Conedera \& Ghiringhelli 2014). Unter den von Menschen verursachten Bränden sind die meisten (37.2\%) durch Fahrlässigkeit entstanden. In 9.8\% der Fälle ist Brandstiftung die Ursache. Brandstiftung ist sehr problematisch, da die Brände meist bei höchster Waldbrandgefahr gelegt werden und die Brandstifter oft mehr als ein Feuer entzünden. Die Brände führen dann oft zu sehr schwer kontrollierbaren Ereignissen. Bei einem weiteren Drittel bleibt die Ursache unbekannt.

Für das Waldbrandmanagement ist im Kanton Tessin grundsätzlich der Forstdienst verantwortlich. Er ist zuständig für die Definition der strategischen Leitlinien im Waldbrandmanagement, die Wald-

1 www.wsl.ch/swissfire (25.3.2019) 


\begin{tabular}{|c|c|c|}
\hline \multirow[t]{2}{*}{ Handlungsfeld } & \multicolumn{2}{|c|}{ Zeithorizont } \\
\hline & kurzfristig & langfristig \\
\hline \multirow[t]{3}{*}{ Prävention } & $\begin{array}{l}\text { Einschätzung der Brandgefahr: } \\
\text { - Fire Niche } \\
\text { - Fire Less } 2 \\
\text { - Fachgruppe Walbbrand } \\
\text { des Forstdienstes }\end{array}$ & $\begin{array}{l}\text { Sensibilisierung der } \\
\text { Gesellschaft }\end{array}$ \\
\hline & $\begin{array}{l}\text { Veröffentlichung der } \\
\text { Waldbrandgefahrenstufe } \\
\text { (BAFU) }\end{array}$ & \multirow[t]{2}{*}{$\begin{array}{l}\text { Waldbau, Biomasse- } \\
\text { regulierung in Risiko- } \\
\text { beständen }\end{array}$} \\
\hline & Absolutes Feuerverbot & \\
\hline \multirow[t]{4}{*}{$\begin{array}{l}\text { Technische und } \\
\text { organisatorische } \\
\text { Massnahmen }\end{array}$} & \multirow[t]{4}{*}{$\begin{array}{l}\text { Pikettdienste: } \\
\text { - Feuerwehr } \\
\text { - Helikopter } \\
\text { - Forstdienst }\end{array}$} & $\begin{array}{l}\text { Löschinfrastruktur: } \\
\text { - Löschwasserbecken } \\
\text { - Hydrantennetz } \\
\text { - Strassennetz }\end{array}$ \\
\hline & & $\begin{array}{l}\text { Waldbrandspezifische Geräte } \\
\text { und Fahrzeuge }\end{array}$ \\
\hline & & $\begin{array}{l}\text { Feuerwehrorganisation } \\
\text { (Bergfeuerwehr) }\end{array}$ \\
\hline & & $\begin{array}{l}\text { Aus- und Weiterbildung von } \\
\text { Feuerwehr, Forstpersonal, } \\
\text { Personal der Helikopterfirmen }\end{array}$ \\
\hline \multirow[t]{3}{*}{ Löschaktion } & $\begin{array}{l}\text { Kantonale Alarmzentrale } \\
\text { (CECAL) }\end{array}$ & Übungen zur Löschtaktik \\
\hline & \multirow{2}{*}{$\begin{array}{l}\text { Gestaffeltes Aufgebot von } \\
\text { Feuerwehr (Stadtfeuerwehr } \\
\text { und, bei Bedarf, auch } \\
\text { Bergfeuerwehr) }\end{array}$} & $\begin{array}{l}\text { Prüfung der Funktions- } \\
\text { fähigkeit der Infrastrukturen }\end{array}$ \\
\hline & & $\begin{array}{l}\text { Kritische Nachbearbeitung } \\
\text { des Einsatzes }\end{array}$ \\
\hline \multirow[t]{3}{*}{$\begin{array}{l}\text { Post-Brand- } \\
\text { Management }\end{array}$} & $\begin{array}{l}\text { Waldbranderfassung in der } \\
\text { Datenbank «Swissfire» }\end{array}$ & \multirow{3}{*}{$\begin{array}{l}\text { Waldbauliche Wieder- } \\
\text { instandstellungsprojekte }\end{array}$} \\
\hline & $\begin{array}{l}\text { Einschätzung der Brand- } \\
\text { schwere und der } \\
\text { Bestandesresilienz }\end{array}$ & \\
\hline & $\begin{array}{l}\text { Temporäre technische } \\
\text { Schutzmassnahmen }\end{array}$ & \\
\hline
\end{tabular}

Massnahmen

$\square$ vor, $\square$ während, $\square$ nach dem Waldbrand

Abb 2 Schematische Darstellung des Konzepts «Waldbrand 2020» des Kantons Tessin.

brandprävention sowie die Beratung der lokalen Behörden bei der Waldbewirtschaftung und der Planung von Löscheinrichtungen. Darüber hinaus definiert er die Regeln für den Löscheinsatz per Helikopter und erfasst die Waldbrände für die Statistik (Conedera et al 2004). Die eigentliche Waldbrandbekämpfung ist Aufgabe der Feuerwehr, der Forstdienst steht den Einsatzkräften aber beratend zur Seite.

\section{Entstehung des kantonalen Konzepts «Waldbrand 2020»}

Das Konzept «Waldbrand 2020» (SFC 2017) ist das Resultat einer intensiven Analyse, Diskussion und Synthesearbeit unter der Federführung des kantonalen Forstdienstes und in enger Zusammenarbeit mit dem kantonalen Feuerwehrverband (Federazione Cantonale Ticinese dei Corpi Pompieri - FCTCP) und der Eidgenössischen Forschungsanstalt für Wald, Schnee und Landschaft (WSL). Datengrund- lage für die Situationsanalyse bildeten die Angaben zur Feuerwehrorganisation und die Waldbrandstatistik aus der Datenbank «Swissfire» (Pezzatti et al 2019, dieses Heft). Beigezogen wurden zudem Modelle und Methoden, die die Forschung im Gebiet entwickelt hatte, zum Beispiel ein Modell zur Vorhersage menschbedingter Brandausbrüche (Wildland-Urban Interface; Conedera et al 2015), Methoden zur Vorhersage der aktuellen Waldbrandgefahr (Pezzatti et al 2015) sowie die Waldbrandrisikokarte (Conedera 2009). Letztere ist von zentraler Bedeutung, da der Umgang mit Risiken und deren Verminderung tragende Säulen des Konzepts «Waldbrand 2020» sind. Risiko ist dabei definiert als die Kombination von Gefahr (Wahrscheinlichkeit eines Waldbrandes) und Gebietsanfälligkeit (Auswirkungen eines Waldbrandes auf die Infrastrukturen und Ökosysteme).

Das Konzept besteht aus vier Handlungsfeldern, die eng ineinander verwoben sind: 1) der Prävention, 2) den technischen und organisatorischen Massnahmen, 3) der Löschaktion und 4) dem PostBrand-Management. Dabei ist jedes Handlungsfeld unentbehrlich, damit das Konzept aufgeht. Die einzelnen Handlungsfelder bestehen je nach Zielsetzung sowohl aus kurzfristigen als auch aus langfristigen Massnahmen zur Verminderung des Waldbrandrisikos (Abbildung 2).

\section{Prävention}

Langfristig ausgerichtete Präventionsmassnahmen beinhalten generell Aktivitäten, mit denen die Bevölkerung, insbesondere die Schulkinder, für einen sorgsamen Umgang mit der Umwelt sensibilisiert und auf die Wichtigkeit der Schutzfunktion der Wälder hingewiesen wird. Das Waldbrandrisiko kann langfristig auch durch waldbauliche Massnahmen reduziert werden, zum Beispiel durch die Anpassung der Baumartenzusammensetzung oder durch gezielte Eingriffe in die krautige und verholzte Vegetation in besonders waldbrandgefährdeten Gebieten (z.B. Entfernen von Nekromasse oder Niederhalten der Unterschicht, um Kronenbrände zu verhindern; Abbildung 3).

Kurzfristige Präventionsmassnahmen sind vor allem im Fall von akuter Brandgefahr wichtig. Sie bestehen im Wesentlichen darin, ein absolutes Feuerverbot auszusprechen, um Brandausbrüche zu verhindern. Für die Einschätzung werden drei verschiedene Ansätze kombiniert, was auch erlaubt, die Resultate der einzelnen Ansätze zu kontrollieren:

1. das neu entwickelte Modell «FireNiche», das mithilfe von Daten aus der Waldbrandstatistik und von meteorologischen Angaben einer repräsentativen Wetterstation die tägliche Waldbrandgefahr für eine bestimmte Region vorhersagt, 

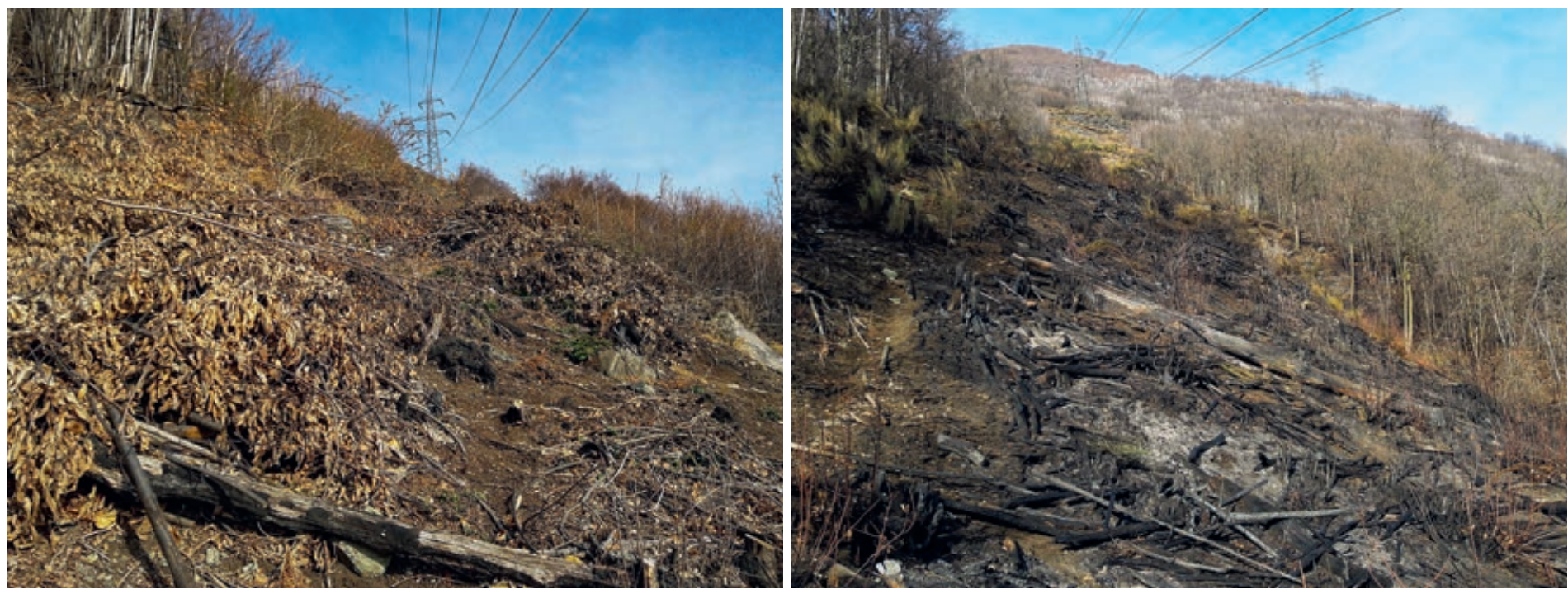

Abb 3 Beispiel eines waldbrandbegünstigenden Umgangs mit Brandgut: Biomasse aus dem Unterhalt von Hochspannungsleitungen vor (links) und nach einem Brand (rechts). Fotos: Marco Conedera

2. kabellose, automatische Bodenfeuchtesensoren, die Feuchtigkeitswerte von Brandgut wie Streu und Humus im Wald erfassen und in Echtzeit übertragen (Fire Less 2), und

3. die herkömmliche, gutachterliche Gefahrenabschätzung im Feld durch die Fachgruppe «Waldbrand», die aus zehn Fachleuten (Forstingenieure und Förster) aus dem kantonalen Forstdienst besteht.

Damit soll die lokale Waldbrandgefahr zeitund fachgerecht ermittelt und die Alarmbereitschaft der Feuerwehr situationsgerecht festgelegt werden (Pezzatti et al 2015). Daraus abgeleitet wird auch die Gefahrenstufe nach der fünfstufigen Skala der Verordnung über die Warnung, die Alarmierung und das Sicherheitsfunknetz der Schweiz (VWAS; SR 520.12).

Die Gefahrenstufe wird separat für die drei kantonalen Untergebiete Sottoceneri, Ticino Centrale und Alto Ticino erstellt und dem Bundesamt für Umwelt (BAFU) zur Publikation auf der offiziellen Website ${ }^{2}$ weitergeleitet. Die Unterteilung in drei Unterregionen stellt einen Kompromiss zwischen einer möglichst realitätsgetreuen Darstellung der Situation für die Fachleute (Forstdienst, Feuerwehr, Kantonspolizei) und einer möglichst einfachen und einheitlichen Kommunikation nach aussen dar. Hauptinstrument für die Kommunikation mit der Bevölkerung ist der Erlass eines absoluten Feuerverbots im Freien, eine Massnahme, die ursprünglich auf einer rechtlichen Grundlage von 1975 basiert, vom Forstdienst in eigener Verantwortung entschieden werden kann und so schnell und einheitlich im ganzen Kanton umgesetzt werden kann (Conedera et al 2004). Über das absolute Feuerverbot wird dann über offizielle Kanäle wie Fernsehen, Radio, Internet und Newsletter informiert, damit möglichst viele Brände menschlicher Ursache vermieden werden können.

\section{Technische und organisatorische Massnahmen}

Langfristig ausgerichtete Massnahmen im Handlungsfeld der technischen und organisatorischen Massnahmen (Abbildung 2) zielen einerseits auf die Löschinfrastrukturen und andererseits auf die Organisation und Koordination aller in die Brandbekämpfung involvierten Dienste und Fachstellen $\mathrm{ab}$

Die Planung, die Realisierung und der Unterhalt der Löschinfrastruktur, insbesondere der Wasserbecken für Löscheinsätze mit Helikopter sowie der Hydrantennetze und der natürlichen Wasserstellen für die Brandbekämpfung vom Boden aus, sind sicher die aufwendigsten Aktivitäten. Dank den Löschwasserbecken in besonders brandgefährdeten Gebieten lassen sich die Rotationszeiten der Helikopter wesentlich reduzieren und so die Löschaktionen effizienter machen. Die von Bund und Kanton subventionierten Löschinfrastrukturen werden gemäss den Prioritäten, die in der Waldbrandrisikokarte ausgewiesen sind, von den lokalen Behörden realisiert (Conedera 2009; Abbildung 4). Eine weitere wichtige technische Massnahme betrifft die laufende Erneuerung, die Ergänzung und den Unterhalt des waldbrandspezifischen Fahrzeug- und Geräteparks.

Die Feuerwehr wurde im Kanton Tessin in jüngerer Vergangenheit mehrmals reorganisiert, um Doppelspurigkeiten zu vermeiden und sie an die technischen (z.B. neue Geräte und Technologien) und gesellschaftlichen Entwicklungen (z.B. zunehmende Distanz zwischen Wohn- und Arbeitsort) anzupassen. Die grösste Reorganisation fand im Jahr 2003 statt, als die Zahl der Feuerwehreinheiten von

2 www.waldbrandgefahr.ch (25.3.2019) 


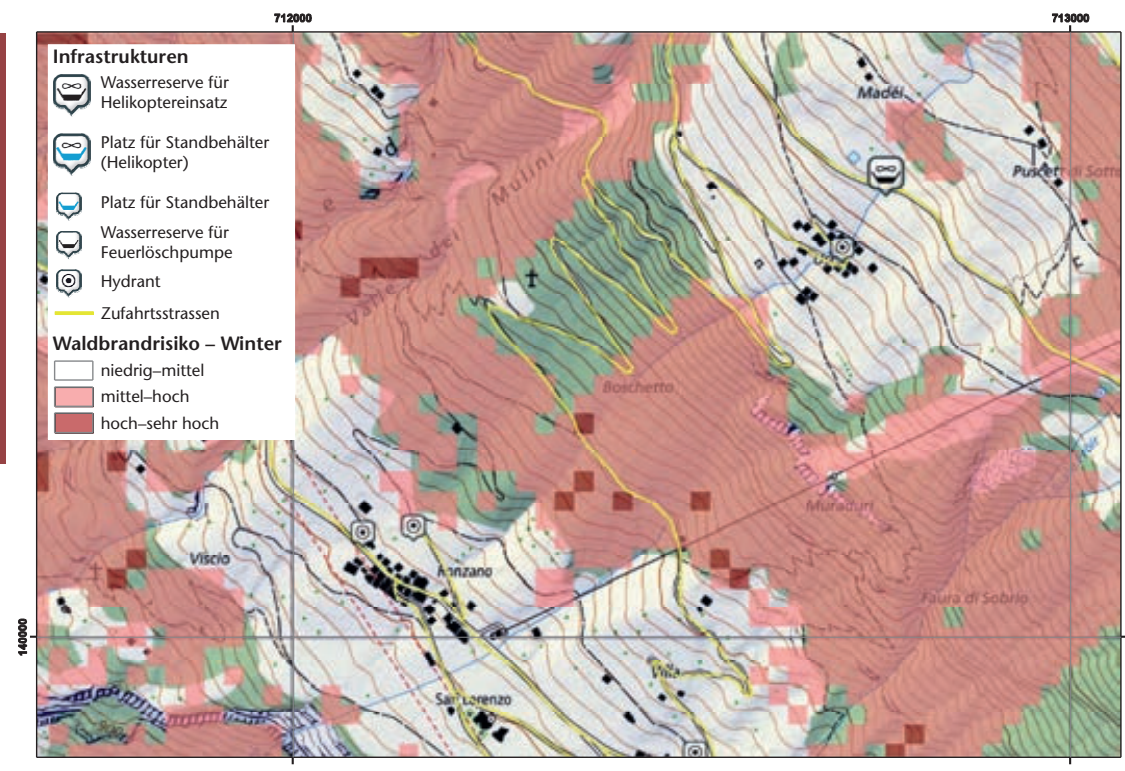

Abb 4 Auszug aus der Waldbrandrisikokarte mit Angabe der Löschwasser-Entnahmestellen. Quelle: Ufficio Forestale $1^{\circ}$ circondario, Faido

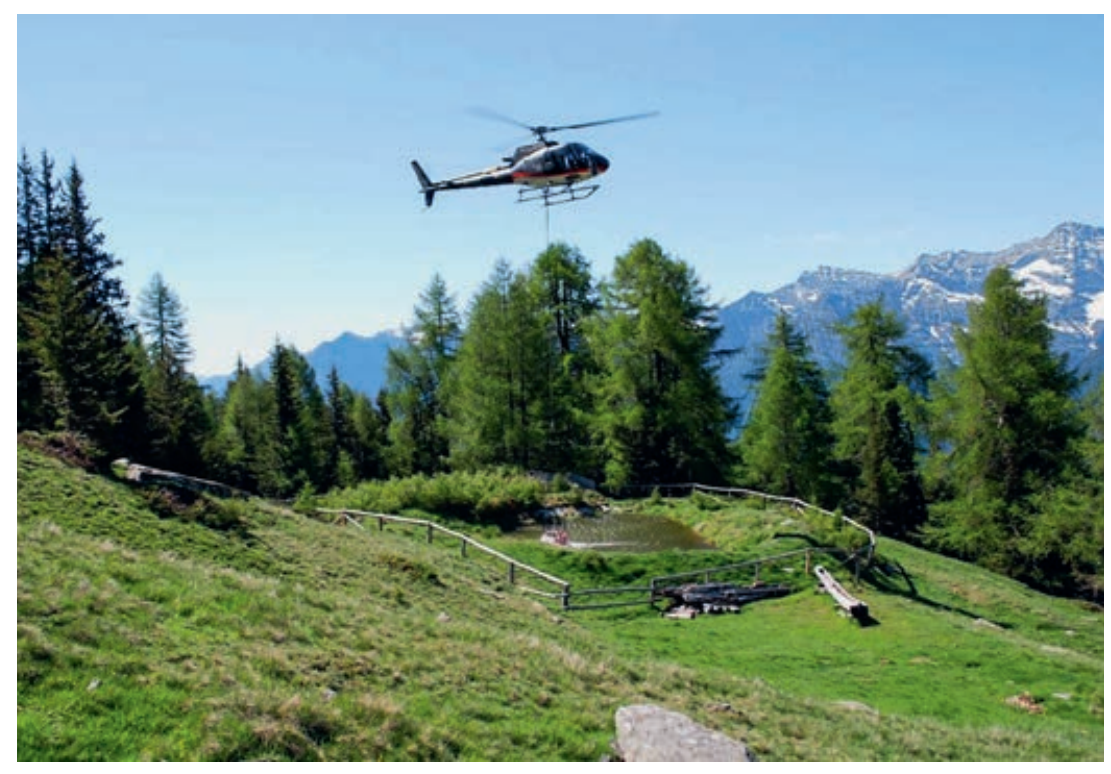

Abb 5 Prüfung der Zweckmässigkeit und der Funktionalität eines Helikopter-Löschwasserbeckens. Foto: Corpo Pompieri Faido

115 auf 31 reduziert wurde (Calabresi 2005, FCTCP 2015). Im Zuge von Gemeindefusionen dürften die Feuerwehreinheiten in nächster Zukunft weiter reduziert werden.

Tragende Säule der heutigen Feuerwehrorganisation ist die Unterteilung in eine Stadtfeuerwehr und eine Bergfeuerwehr. Die Stadtfeuerwehr besteht aus 1134 Mitgliedern (davon 28 vollberufliche Feuerwehrleute) und ist hierarchisch und dezentral in 5 überregionale Hauptzentren, 15 regionale und 5 lokale Zentren organisiert. Diese Struktur garantiert eine gute Präsenz auf dem ganzen Territorium und eine rasche Einsatzbereitschaft auch bei Waldbrandmeldungen. Bei der Bergfeuerwehr handelt es sich um auf Waldbrände spezialisierte Einheiten, die Anfang der 1980er-Jahre in den von Waldbränden am stärksten betroffenen Gebieten gegründet worden sind. Sie zählt heutzutage 317 Milizpersonen (von denen 37 auch in der Stadtfeuerwehr sind). Geglie- dert ist die Bergfeuerwehr in drei regionale Einheiten sowie in acht in die Stadtfeuerwehr integrierte Sektionen. Im Notfall, d.h. im Fall von grossen Bränden oder Brandausbrüchen an verschiedenen Orten, kann die Feuerwehr durch weitere Dienste verstärkt werden, insbesondere aus Beständen des Zivilschutzes und der Armee. So muss die Feuerwehr in ihrem Bestand nicht auf seltene und extreme Brandsituationen dimensioniert werden.

Für die Löschaktionen aus der Luft werden private Helikopterfirmen aufgeboten. Die Grundsätze der Zusammenarbeit (Anzahl Helikopter und Ausrüstungstyp, Reaktionszeit nach Alarmierung, Tarife) sind in einer Vereinbarung zwischen dem Tessiner Umweltdepartement und den privaten Helikopterfirmen geregelt. Die Vereinbarung wurde Anfang 2019 revidiert mit dem Ziel, die Einsatzbereitschaft, die Koordination und die Sicherheit im Ereignisfall zu verbessern. Nur ausnahmsweise werden für Löschaktionen auch Armeehelikopter beigezogen. Deren Einsatz richtet sich nach dem Prinzip der Subsidiarität und ist unabhängig von der mit den privaten Firmen getroffenen Vereinbarung.

Die Aus- und Weiterbildung ist eine Daueraufgabe bei der Feuerwehr. Übungen, in die alle in die Waldbrandbekämpfung involvierten Dienste eingebunden sind, sind besonders nützlich, um die Löschtaktik und die Abstimmung zwischen den verschiedenen Akteuren zu trainieren. Die Übungen dienen gleichzeitig auch dem Testen der Tauglichkeit und der Funktionsfähigkeit der Löschinfrastruktur (Strassen- und Hydrantennetze, Löschwasserbecken; Abbildung 5). Die im Jahr 2015 im Forstdienst geschaffene Fachgruppe «Waldbrand» beschäftigt sich vor allem mit Fragen der Waldbrandprävention, der Löschinfrastruktur und der Organisation im Ereignisfall und ist seit 2019 auch im Ausbildungsbereich aktiv. Einerseits bildet sie einzelne Mitarbeiter des Forstdienstes zu Waldbrandspezialisten aus, damit diese die Einsatzleiter während der Löscheinsätze fachlich beraten können. Andererseits ist sie an der Ausbildung der angehenden Feuerwehrleute beteiligt und unterrichtet dort das waldspezifische Wissen.

Sehr wichtig ist auch die kritische Nachbearbeitung der Löschaktionen (Abbildung 6), um Schwachpunkte zu erkennen und Korrekturmassnahmen zu implementieren. Beispielhaft in diesem Zusammenhang ist die Tagung, die nach dem Trockensommer 2013 organisiert wurde, um den Erfahrungsaustausch im Umgang mit Blitzschlagbränden zu ermöglichen (Conedera \& Ghiringhelli 2014). Dieser Erfahrungsaustausch hat zu einer Verbesserung sowohl des Löschmaterials (z.B. helikoptertaugliche Hochdruckgeräte und geschlossene Wasserbehälter) als auch der Einsatzstrategie geführt. Auch der Ansatz zur Einschätzung der Gefahr von Blitzschlagbränden wurde angepasst und basiert nun nur noch auf der Anzahl Ereignisse und nicht mehr auch 


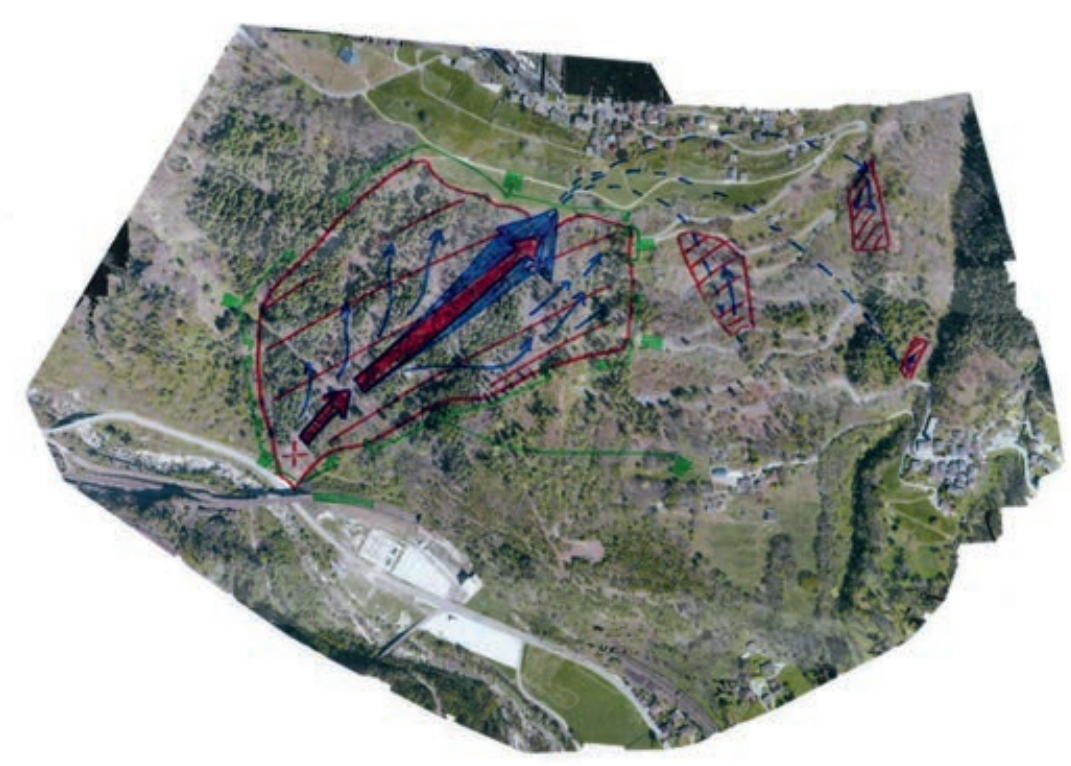

Abb 6 Waldbrand vom April 2017 in Osco (Leventina). Die Feuerfront und die daraus resultierende Thermik waren derart intensiv, dass bis in eine Distanz von $300 \mathrm{~m}$ neue Brandherde durch Funkenflug entstanden sind. Foto: Oblivion Aerial SA

auf deren Ausdehnung, die eher von der Zugänglichkeit der Brandfläche abhängt.

Kurzfristige Massnahmen im Bereich der Organisation betreffen im Wesentlichen den Pikettdienst der in die Löschaktionen involvierten Stellen. Im Fall der Aktivierung des absoluten Feuerverbots wird der Bereitschaftsgrad des Forstpersonals und der Helikopterfirmen durch den Forstdienst bestimmt. Die Feuerwehr bestimmt ihren Bereitschaftsgrad selbst, wobei derjenige der Bergfeuerwehr sich beim absoluten Feuerverbot automatisch erhöht.

\section{Löschaktion}

Zweck der Löscharbeiten ist es nicht nur, die durch Brände direkt verursachten Personen- und Sachschäden möglichst gering zu halten, sondern auch Folgeschäden vorzubeugen, die durch Erosion, Murgang oder Steinschlag entstehen könnten. Die Grösse der Brandfläche ist in diesem Zusammenhang entscheidend, da kleine Brände die Schutzfunktion des Waldes selten beeinträchtigen. Grosse Brandflächen können vermieden werden, wenn dank einem guten Einsatzdispositiv die Reaktionszeit kurz ist und wenn die richtige Löschtaktik und die richtigen Löschmittel zum Einsatz kommen.

Die Fähigkeit, den Bereitschaftsgrad flexibel an die Gefahrensituation anzupassen und unmittelbar auf Brandmeldungen zu reagieren, ist die wichtigste kurzfristige Massnahme. Ein grosser Fortschritt diesbezüglich war die Einrichtung einer kantonalen Alarmzentrale (CECAL118), die alle Feuermeldungen direkt verwaltet und die den zum Waldbrand nächst gelegenen Feuerwehrstützpunkt sowie den Forstdienst aktiviert.
Bei den Löscharbeiten hat der Forstdienst beratende Funktion. In der ersten Löschphase ist es vor allem der Revierförster, der dank seinen Lokalkenntnissen den Einsatzleiter berät: Wie steht es um die Walderschliessung und deren Befahrbarkeit, wo sind Holzschläge im Gang? Wer von den örtlichen Behörden ist zu informieren, welche Waldbesitzer sind betroffen? Befinden sich Infrastrukturen im Gebiet, auf die der Brand keinesfalls übergreifen darf, und wo liegen die Schutzwälder? Eine zweite Stufe der Beratung, die eher vom auf Waldbrand spezialisierten Forstpersonal übernommen wird, betrifft die Einschätzung der Gefahrensituation und des Feuerverhaltens. Diese Einschätzung kann dazu führen, dass der Einsatzleiter die Löschtaktik oder die Löschprioritäten ändert. Normalerweise fällt der Einsatzleiter auch den Entscheid, Spezialgeräte (z.B. Militärhelikopter) beizuziehen, in Absprache mit dem spezialisierten Forstpersonal.

\section{Post-Brand-Management}

Der erste Schritt im Handlungsfeld des PostBrand-Managements besteht darin, dass der zuständige Revierförster die Brandfläche besucht und alle für die Beschreibung des Ereignisses erforderlichen Angaben erfasst. Dazu gehören insbesondere die Brandursache, der Brandperimeter, der Waldtyp, die Hauptbaumart sowie der Umfang der Schäden. In einem zweiten Schritt klärt er zusammen mit dem Kreisförster, wie stark die Schutzwirkung des Waldes durch das Feuer reduziert worden ist, welche Folgen dies für Menschenleben und Sachwerte hat und wie rasch sich die betroffene Fläche erholen kann (Conedera et al 2010). Problematisch auf Brandflächen sind vor allem der stärkere Oberflächenabfluss und die höhere Erosionsanfälligkeit (Providoli et al 2002), die je nachdem zu Murgängen führen können (Conedera et al 2003).

Wenn die Schutzfunktion mittel- bis langfristig beeinträchtigt ist (siehe z.B. Maringer et al 2016), müssen technische (z.B. temporäre Verbauungen) und waldbauliche Massnahmen (z.B. Aufforstung) getroffen werden, um die drohenden Gefahren einzudämmen und die Erholungszeit des Waldes zu verkürzen (Frehner \& Plozza 2018; Abbildung 7). Langfristig sollten die betroffenen Waldflächen so bewirtschaftet werden, dass deren Resistenz und Resilienz gegenüber den Effekten von Feuer und Klimaveränderungen steigen.

\section{Erste Evaluation des kantonalen Konzepts «Waldbrand 2020»}

Im Konzept «Waldbrand 2020» (SFC 2017) sind die für den ganzen Kanton Tessin geltenden 

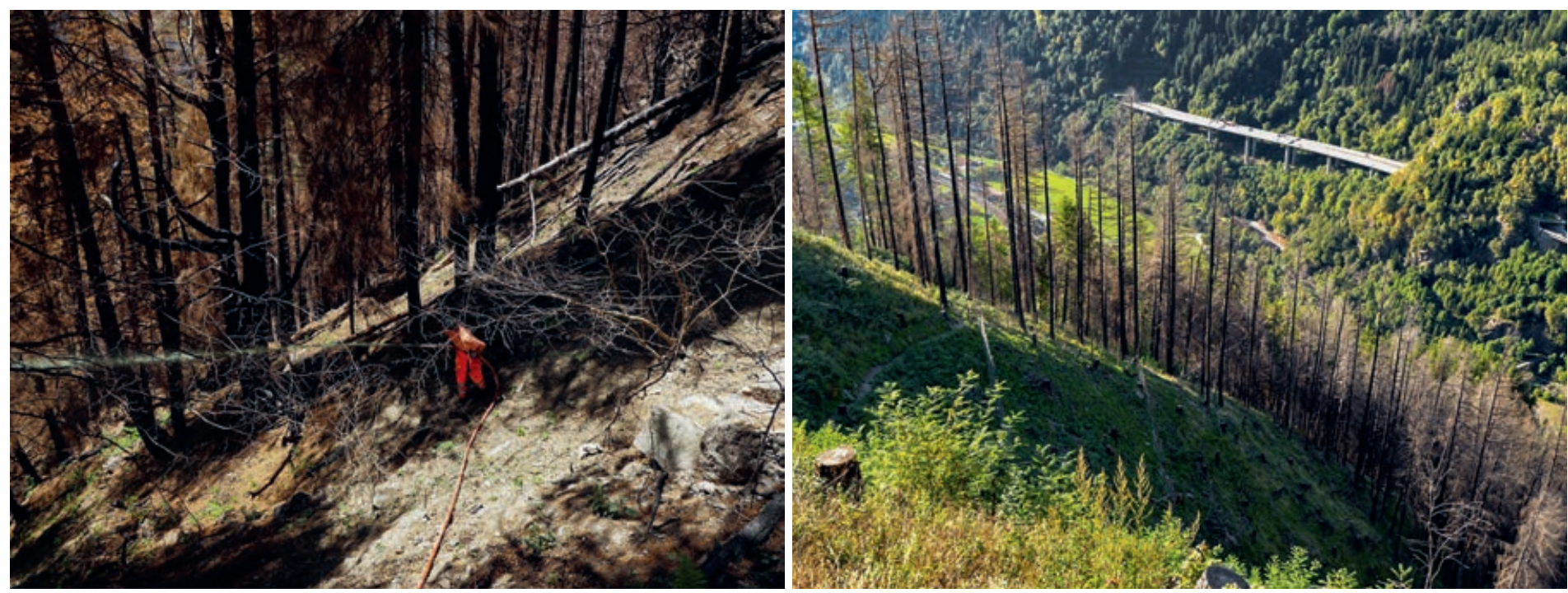

Abb 7 Waldwiederinstandstellung nach dem Waldbrand vom April 2017 in Osco (Leventina). Die ursprünglich gepflanzten Fichten, Lärchen und Föhren wurden vom Kronenfeuer total zerstört (Bild links). Teilweise zerstört wurde auch der Rohhumus. Um die Erosion zu bekämpfen und den neuen Waldbestand zu fördern, wurde mittels Hydrosaat eine Mischung aus ausgewählten Krautpflanzen und Baumarten ausgebracht (Bild links), sodass nach wenigen Wochen bereits ein grüner Erosionsschutz vorhanden war (Bild rechts). In einer zweiten Phase werden in durch Wildzäune geschützten Teilflächen Waldbäumchen angepflanzt. Fotos: Roberto Albertini (links) und Aron Ghiringhelli (rechts)

Grundsätze und Ziele des Waldbrandmanagements verankert. Dies ermöglicht es nicht nur, die kurzund langfristigen Massnahmen überall nach dem gleichen Standard zu planen, sondern auch deren Erfolg zu evaluieren und Verbesserungen zu implementieren. Eine solche Erfolgskontrolle ist unabdingbare Voraussetzung für das Funktionieren einer solch komplexen Organisation, an der Behörden aller Stufen (Gemeinden, Kanton, Bund) und Akteure verschiedenster Art (Beamte, Freiwillige, private Firmen) beteiligt sind. Das Konzept ist erst zwei Jahre in Kraft, und es sind noch nicht alle vorgesehenen Massnahmen implementiert. Dennoch soll versucht werden, anhand der bisherigen Erfahrungen eine erste kritische Evaluation durchzuführen.

Die Methoden und Technologien zur Ermittlung der täglichen Brandgefahr (Fire Niche, Fire Less 2, Fachgruppe Waldbrand des Forstdienstes) haben sich in den Trockenperioden, die über die Jahreswechsel 2016/2017 und 2018/2019 herrschten, bewährt. Es hat sich aber gezeigt, dass in den Entscheid, ob ein absolutes Feuerverbot im Freien erlassen werden soll, auch weitere, «weiche» Faktoren einbezogen werden müssen. So sind zum Beispiel an den Wochenenden oder über die Feiertage viel mehr Leute unterwegs. Dass das absolute Feuerverbot damals unterschiedslos für den ganzen Kanton ausgesprochen wurde, stellte sich als eine sehr effiziente Massnahme heraus. Einerseits wurde es von der örtlichen Bevölkerung und den Touristen einfacher respektiert, andererseits konnte die Reaktionszeit der Feuerwehr erheblich reduziert werden, weil nun jede Rauchsäule potenziell ein Waldbrand hätte sein können.

Die Organisation zur Bekämpfung von Waldbränden hat sich in den letzten zwei Jahren im
Grundsatz auch bewährt. Sie hat aber noch Verbesserungspotenzial. Bei 31 der 40 Waldbrände konnte die Fläche unter der Schwelle von einer Hektare gehalten werden. Bei Ereignissen, bei denen das Feuer trotz Löscheinsatz auf weitere Flächen übergriff, zeigte sich, wie wichtig das Vorhandensein von Löschwasserbecken in der entsprechenden Höhenlage ist. Denn kurze Rotationszeiten erhöhten die Löscheffizienz der Helikopter markant. Für viele wichtige Schutzwälder muss das Löschbeckennetz noch vervollständigt werden.

Die Arbeit der Feuerwehr am Boden bleibt entscheidend und muss ständig mit dem Helikoptereinsatz koordiniert werden. Einerseits können die Leute im Feld die Prioritäten für die Löschaktionen aus der Luft am besten erkennen, anderseits können sie die verbleibenden Brandherde (brennende Stöcke, unterirdische Schwelfeuerstellen) direkt löschen. Auch in diesem Bereich gibt es Verbesserungsmöglichkeiten, vor allem auf der Ebene der spezifischen Fachkenntnisse und Löschtechniken, damit solch komplexe Vorgänge während des Einsatzes reibungslos funktionieren. Die Ausbildung aller Akteure ist auch hier zentral. Seit diesem Jahr gibt es eine gezielte und vertiefte Ausbildung auch für das Personal der Helikopterfirmen.

Dass die Stadtfeuerwehr bei Waldbränden zuerst ausrückt und die Bergfeuerwehr ihr bei anspruchsvollen Bränden zu Hilfe kommt, ist mehrheitlich eine gute Lösung. Allerdings kann es zu gravierenden Engpässen kommen, falls die Stadtfeuerwehr am Löschen eines Waldbrands ist und sich gleichzeitig auch noch andere Brände oder Unfälle ereignen. Die Bergfeuerwehr ist dagegen voll auf die Waldbrandbekämpfung fokussiert und sam- 
melt dort die nötige Erfahrung, um die Löschaktionen immer besser und effizienter abzuwickeln. Für die Zukunft ist daher geplant, die Bergfeuerwehr rascher aufzubieten und sie koordiniert im ganzen Kanton einzusetzen. Die im Konzept «Waldbrand 2020» vorgesehene Aufwertung und Verstärkung der Bergfeuerwehr ist in diesem Sinn ein sehr wichtiger Schritt.

Ein weiterer verbesserungswürdiger Punkt betrifft die Aus- und Weiterbildung der Einsatzleiter bei der Feuerwehr und des beratenden Forstpersonals, damit bei jedem Einsatz ein sehr hoher Qualitätsstandard garantiert werden kann, unabhängig von der effektiven Erfahrung der jeweiligen Person. Heutzutage sind die Brandereignisse allzu spärlich, als dass sich alle Einsatzleiter genügend Felderfahrung aneignen könnten. Für die Zukunft wäre daher eine weitere Spezialisierung in dieser Schlüsselfunktion angezeigt.

\section{Schlussbemerkungen}

Die Waldbrandmanagementstrategie des Kantons Tessin wurde vor zwei Jahren im von allen beteiligten Institutionen mitgetragenen Konzept «Waldbrand 2020» festgelegt, mit dem Ziel, das Waldbrandmanagement auf kantonaler Ebene einheitlich und nach klaren Prioritäten durchzuführen und Waldbrände koordiniert, professionell, effizient und mit angepasstem Mitteleinsatz zu bekämpfen. Waldbrände werden nie komplett verhindert werden können. Blitzschlagbrände wird es immer geben, und die menschlichen Aktivitäten lassen sich nie vollständig kontrollieren. Daher ist die Löschaktion unbestritten ein sehr wichtiges Handlungsfeld. Genauso wichtig sind aber auch die anderen Handlungsfelder - die Prävention, die organisatorischen und technischen Massnahmen sowie das Post-Brand-Management, um die von Waldbränden ausgehenden Risiken angesichts der sich verändernden klimatischen und sozioökonomischen Bedingungen unter Kontrolle halten zu können.

Eingereicht: 4. Februar 2019, akzeptiert (mit Review): 20. Mai 2019

\section{Literatur}

CALABRESI A (2005) L'organizzazione pompieristica cantonale. Dati statistiche e società 5 (1): 43-46.

CESCHI I (1975/76) Gli incendi boschivi in Canton Ticino. Boll Soc ticin Sci nat 65: 63-102.

CESTI G (1990) II vento e gli incendi boschivi. Indagine sulla ventosità invernale in Valle d'Aosta. Châtillon: Regione Autonoma Valle d'Aosta. 159 p.

CIOLDI F, BALTENSWEILER A, BRÄNDLI UB, DUC P, GINZLER C ET AL (2010) Waldressourcen. In: Brändli UB, editor. Schweizerisches Landesforstinventar. Ergebnisse der dritten Erhebung 2004-2006. Birmensdorf: Eidgenöss Forsch.anstalt WSL. pp. 31-113.
CONEDERA M, MARCOZZI M, JUD B, MANDALLAZ D, CHATELAIN F ET AL (1996) Incendi boschivi al Sud delle Alpi: passato, presente e possibili sviluppi futuri. Zürich: vdf Hochschulverlag. $143 \mathrm{p}$.

CONEDERA M, PETER L, MARXER P, FORSTER F, RICKENMANN D ET AL (2003) Consequences of forest fires on the hydrogeological response of mountain catchments: A case study of the Riale Buffaga, Ticino, Switzerland. Earth Surf Process Landf 28: 117-129.

CONEDERA M, CORTI G, PICCINI P, RYSER D, GUERINI F ET AL (2004) La gestione degli incendi boschivi in Canton Ticino: tentativo di una sintesi storica. Schweiz Z Forstwes 155: $263-$ 277. doi: $10.3188 /$ szf.2004.0263

CONEDERA M, PEZZATTI GB (2005) Gli incendi di bosco: cosa ci dice la statistica. Dati statistiche e società 5 (1): 6-8, 10-13.

CONEDERA M, CESTI G, PEZZATTI GB, ZUMBRUNNEN T, SPINEDI F (2006) Lightning-induced fires in the Alpine region: An increasing problem. In: Viegas DX, editor. Figueira da Foz. Coimbra: University of Coimbra. $9 \mathrm{p}$.

CONEDERA M (2009) Implementing fire history and fire ecology in fire risk assessment: the study case of Canton Ticino (southern Switzerland). Karlsruhe: Univ Fridericiana, PhD-Thesis. $82 \mathrm{p}$.

CONEDERA M, LUCINI L, VALESE E, ASCOLI D, PEZZATTI GB (2010) Fire resistance and vegetative recruitment ability of different deciduous trees species after low- to moderate-intensity surface fires in southern Switzerland. In: Viegas DX, editor. Proc Int Conf Forest fire research, 15-18 November 2010 in Coimbra. $12 \mathrm{p}$.

CONEDERA M, GHIRINGHELLI A (2014) Gli incendi boschivi causati da fulmine: un fenomeno in crescita. Agricoltore ticinese 146: 9.

CONEDERA M, TONINI M, OLEGGINI L, VEGA OROZCO C, LEUENBERGER M ET AL (2015) Geospatial approach for defining the Wildland-Urban Interface in the Alpine environment. Comp Environ Urban Syst 52: 10-20.

FCTCP (2015) Di fuoco e di fatiche. Federazione cantonale ticinese corpi pompieri 75 anni 1940-2015. Pregassona: Fontana. $369 \mathrm{p}$.

FREHNER M, PLOZZA L (2018) Waldbrand Misox: Pflanzungen mit Berücksichtigung des Klimawandels. Bündner Wald 71 (6): 20-25.

KOCHER SD, BUTSIC V (2017) Governance of land use planning to reduce fire risk to homes in Mediterranean France and California. Land 6 (2): 24

MARINGER J, ASCOLI D, DORREN L, BEBI P, CONEDERA M (2016) Temporal trends in the protective capacity of burnt beech forests (Fagus sylvatica L.) against rockfall. Eur J For Res 135: 657673.

NCCS, EDITOR (2018) CH2018. Klimaszenarien für die Schweiz. Zürich: National Centre for Climate Services. 24 p.

PEZZATTI GB, ZUMBRUNNEN T, BÜRGI M, AMBROSETTI P, CONEDERA M (2013) Fire regime shifts as a consequence of fire policy and socio-economic development: An analysis based on the change point approach. For Pol Econ 29: 7-18.

PEZZATTI B, DE ANGELIS A, CONEDERA M, GHIRINGHELLI A, BROGGI C ET AL (2015) Tecnologia innovativa e intelligenza artificiale al servizio della prevenzione degli incendi boschivi. In: Federazione cantonale ticinese corpi pompieri, editor. Di fuoco e di fatiche. Federazione cantonale ticinese corpi pompieri 75 anni 1940-2015. Pregassona: Fontana. pp. 361366.

PEZZATTI GB, BERTOGLIATI M, GACHE S, REINHARD M, CONEDERA M (2019) Swissfire: technisch modernisiert und dank Archivrecherchen inhaltlich erweitert. Schweiz Z Forstwes 170: 234-241. doi: 10.3188/szf.2019.0234

PLANAT (2018) Umgang mit Risiken aus Naturgefahren. Bern: Planat. $30 \mathrm{p}$. 
PROVIDOLI I, ELSENBEeR H, CONEDERA M (2002) Post-fire management and splash erosion in a chestnut coppice in southern Switzerland. For Ecol Manage 162: 219-229.

SFC (2007) Piano forestale cantonale. Bellinzona: Sezione forestale cantonale. $42 \mathrm{p}$.

\section{Le programme «Incendie de forêts 2020» du canton du Tessin}

La longue expérience dans la lutte contre les incendies du canton du Tessin a permis le développement d'un effort de compilation, mais aussi d'analyses de ces événements. Ainsi, la gestion des feux de forêt est constamment réexaminée et améliorée dans le but de préserver la forêt de protection et de faire des montagnes un espace sûr pour les habitants. Les améliorations dans l'organisation et les techniques de lutte contre les incendies (par exemple, l'utilisation systématique des hélicoptères comme appui aérien) et la possibilité d'interdire de faire du feu à l'air libre ont permis de réduire le nombre de feux de forêt depuis les années 70 dans le Tessin. Toutefois, à cause du changement climatique et de la demande toujours croissante de protection des personnes et des biens dans nos sociétés modernes, le risque d'incendie pourrait s'accroître dans les années à venir. C'est pourquoi le service forestier du Tessin a lancé, il y a deux ans, le programme «Incendie de forêts 2020», en collaboration avec la fédération cantonale des brigades de sapeurs-pompiers et I'Institut fédéral de recherches WSL. Ce programme est composé des quatre parties interdépendantes suivantes: 1) prévention, 2) aspects techniques et organisationnels, 3) contrôle et lutte contre les incendies, 4 ) restauration des parcelles brûlées. Le service forestier assure l'évaluation du danger d'incendie, la publication des interdictions de feu, le conseil envers les autorités locales en matière de gestion forestière et la création d'infrastructures de lutte (comme des points d'eau pour les hélicoptères). II a également pour rôle de définir les règles de lutte aérienne, d'enregistrer les données des incendies pour la statistique et de planifier les mesures de restauration post-incendie. Les pompiers ont, quant à eux, pour mission de contrôler les feux de forêt. Bien que ces derniers soient tout d'abord gérés par les brigades urbaines, un appui des sapeurs-pompiers spécialisés dans la lutte en forêt peut être nécessaire, notamment dans le cas d'incendies étendus. Durant les luttes anti-incendies, le service forestier prend le rôle de consultant. Les deux premières années de mise en service ont permis de montrer la grande pertinence du programme «Incendie de forêts 2020». De possibles améliorations ont toutefois été détectées et sont actuellement en exécution, notamment l'achèvement de la construction des infrastructures de prévention, I'amélioration de la coordination entre services de lutte terrestres et aériens ou la consolidation des brigades de sapeurs-pompiers spécialisées en forêt.
SFC (2017) Concetto cantonale incendi di bosco 2020. Bellinzona: Sezione forestale cantonale. $118 \mathrm{p}$.

\section{The "forest fire 2020" program of Canton Ticino}

The Canton of Ticino has a long-lasting experience in facing forest fires. As a result, a tradition in forest fire documentation and analysis exists and the forest fire management approach is continuously reviewed and improved with the aim to preserve the forest protection functions and to keep the mountain areas safe for the inhabitants. The fire regime has been reduced in Ticino since the seventies of last century thanks to improvement of the firefighting organization and fire control techniques (e.g. systematic use of helicopters for the aerial fire control) and the possibility of declaring a total fire ban in the open. However the demand in terms of protection of human lives and goods of the modern society is raising and as consequence of the climate change fire risk may increase in the future. For this reason two years ago the forest service of Canton Ticino developed the "forest fire 2020 " program, in collaboration with the cantonal fire brigades association and the federal research Institute WSL. The program consists of four interdependent activity modules, which are 1) prevention, 2) organizational and technical measures, 3) firefighting and control, 4) burnt area restoration. The forest service is responsible for the fire-danger rating, the fire-ban release, the mentoring of local authorities in forest management questions and for planning pre-suppression facilities (e.g. water points for helicopters). It is also responsible for defining the mission rules for aerial firefighting, for collecting the data for the statistics, and for planning the post-fire forest restoration measures. The fire brigades are in charge of the firefighting tasks, by first intervening with the urban fire brigades and in case of need requiring the support of specialized forest-fire brigades. During the firefighting actions the forest service takes a consulting role. The first two years of implementation confirmed the suitability of the "forest fire 2020" program. Potential improvements have been however detected and are under implementation, such as the completion of the pre-suppression infrastructures, a better coordination between aerial and terrestrial firefighting and the strengthening of the specialized forest-fire brigades. 